\title{
Adipose-derived stem cells (ADSCs) inhibit the expression of anti-apoptosis proteins through up-regulation of ATF4 on breast cancer cells
}

\author{
Jing Ren ${ }^{1}$, Weishu Kong ${ }^{2}$, Feng Lu ${ }^{1}$, Ye Li ${ }^{1}$ \\ ${ }^{1}$ Department of Plastic and Cosmetic Surgery, Nanfang Hospital, Southern Medical University, Guangzhou, China; ${ }^{2} 32023$ Troops, PLA, Dalian, \\ China \\ Contributions: (I) Conception and design: J Ren, Y Li; (II) Administrative support: F Lu, Y Li; (III) Provision of study materials or patients: W Kong; \\ (IV) Collection and assembly of data: Y Li, F Lu; (V) Data analysis and interpretation: All authors; (VI) Manuscript writing: All authors; (VII) Final \\ approval of manuscript: All authors. \\ Correspondence to: Ye Li. Department of Plastic and Cosmetic Surgery, Nanfang Hospital, Southern Medical University, 1838 Guangzhou North \\ Road, Guangzhou 510515, China. Email: liyefimmu@outlook.com.
}

\begin{abstract}
Background: While current basic studies indicate adipose-derived stem cells (ADSCs) can promote cell proliferation, clinical trials have shown no significant difference in breast cancer recurrence rates for patients with or without autologous fat grafting (AFG). In this study we attempted to explore the underlying mechanism for these contradictory results.
\end{abstract}

Methods: ADSCs and umbilical mesenchymal stem cells (UMSCs) were co-cultured with breast cancer cells (MCF-7 and MDA-MB-231), and the cell viability analyzed by CCK-8 cell proliferation assay, TUNEL assay and immunofluorescence assay. In addition, real-time quantitative polymerase chain reaction (RTqPCR) experiments and Western blot analysis were used to detect the mRNA and protein expression of activating transcription factor 4 (ATF4) and its downstream gene (MCL1 \& BCL2), respectively.

Results: Co-cultured ADSCs could promote cell proliferation and cell apoptosis, and up-regulate ATF4 expression both in MCF-7 and MDA-MB-231. While co-cultured UMSCs could only promote cell apoptosis in MCF-7. Interestingly, we found that when co-cultured ADSCs, the expression of MCL1 and BCL2 protein was decreased, even if their mRNA expression was up-regulated both in MCF-7 and MDAMB-231.

Conclusions: Co-cultured ADSCs can up-regulate ATF4 expression, then interfere with the translation process of MCL1 and BCL2 mRNA and induce cell apoptosis. These data provide insight into the safety characteristics of AFG.

Keywords: Breast cancer; adipose-derived stem cells (ADSCs); activating transcription factor 4 (ATF4); apoptosis; fat grafting

Submitted May 07, 2021. Accepted for publication Aug 06, 2021.

doi: 10.21037/atm-21-3746

View this article at: https://dx.doi.org/10.21037/atm-21-3746

\section{Introduction}

Breast cancer is a common malignant tumor in females worldwide (1). While the current standard treatment for breast cancer is radical surgery with or without chemotherapy and radiotherapy, the imperfect cosmetic appearance after operation is not conducive to the physical and mental recovery of patients. This has resulted in an increase in patients seeking autologous fat grafting (AFG) $(2,3)$, which can reconstruct the natural appearance of the breast and decrease the surgical complication of capsular contracture. Fat tissue contains many adipose-derived stem cells (ADSCs), and can promote wound healing and 
angiogenesis, which provide a better graft for patients (4).

Although AFG had been widely used for patients after radical surgery for breast cancer, its safety remains unclear $(5,6)$. Some previous basic studies indicated breast reconstruction with fat tissues increased the risk of tumor recurrence, and ADSCs could secrete cell factors which could enhance cell proliferation and induce drug resistance in cancer cells (7-9). Wang et al. suggested coculturing ADSCs with the MCF-7 cell line could raise the concentration of CXCL1 and CXCL8, promoting cancer cell proliferation, cancer angiogenesis and tumor growth (10). Duong and his colleagues proposed soluble factors secreted by ADSCs could reduce the anti-tumor effectiveness of trastuzumab (11). However, a long-term clinical study containing 587 patients (287 patients for AFG treatment, 300 patients for control), determined that the use of AFG for breast construction was safe. That research indicated that after 5 years of follow-up, only eight and 11 cases of local recurrence were observed in the AFG group and the control group, respectively, indicating no significant difference in the local recurrence rate between the two groups (12). To our knowledge, numerous basic studies have indicated that co-culture with ADSCs could induce breast cancer cells proliferation, but most clinical studies have shown that AFG treatment could not increase the local recurrence rate of breast cancer (13-15). In addition, another basic study confirmed that umbilical mesenchymal stem cells (UMSCs) could inhibit cell proliferation and induce cell apoptosis $(16,17)$. Therefore, we put forward a hypothesis that ADSCs could not only promote cell proliferation, but could also induce cell apoptosis, to explain these conflicting results.

Activating transcription factor 4 (ATF4) possesses a leucine zipper domain which plays an important role in the development of cancer (18). Some previous studies indicated that ATF4 could directly regulate the translation of anti-apoptosis protein, then induce cell apoptosis (19), suggesting ADSCs might induce cell apoptosis through the ATF4-related pathway. Therefore, in this study, we conceived and designed experiments to explore differences in function between ADSCs and UMSCs and detect a possible mechanism to explain the safety of AFG in clinical application through analyzing the expression level of ATF4 and its downstream anti-apoptosis genes (MCL1 and BCL2). We present the following article in accordance with the MDAR reporting checklist (available at https://dx.doi. org/10.21037/atm-21-3746).

\section{Methods}

\section{Materials}

Dulbecco's modified Eagle's medium (DMEM), DMEM/ F12, fetal bovine serum (FBS), phosphate buffered saline (PBS), and $0.25 \%$ trypsin were obtained from Gibco (New York, NY, USA). The primary anti-bodies (BCL2, MCL1, and GAPDH) and secondary anti-bodies were purchased from Proteintech (Chicago, IL, USA).

\section{Cell lines and cell cultivation}

UMSCs were purchased from the cell bank of the Chinese Academy of Sciences (Shanghai, China), and ADSCs were prepared by the authors following liposuction of the subcutaneous adipose tissue. Briefly, approximately $10 \mathrm{~g}$ of adipose tissue was treated with $0.1 \%(\mathrm{w} / \mathrm{v})$ collagenase solution (collagenase type I; Sigma, St. Louis, MO, USA) at $37^{\circ} \mathrm{C}$ for $1 \mathrm{~h}$ and the tissues were filtered through a $100-\mu \mathrm{m}$ mesh filter to remove debris. The filtered cells were centrifuged at $250 \mathrm{~g}$ for $5 \mathrm{~min}$. The bottom was the pellet which was constituted by the stromal vascular fraction (SVF), then collected at the bottom and filtered through a $70-\mu \mathrm{m}$ mesh filter and seeded at 200,000 cells $/ \mathrm{cm}^{2}$ in DMEM supplemented with $20 \%$ of FBS before incubation at the condition of $37{ }^{\circ} \mathrm{C}$ and $5 \% \mathrm{CO}_{2}$. Cells were passaged every 3 days, and only P3-P5 ADSCs were used.

\section{Preparation of ADSCs-related medium (A-CM) and UMSCs-related medium (U-CM)}

A mesenchymal stem cell growth medium (including DMEM/F12 and $10 \%$ FBS) was used to incubate the ADSCs and UMSCs, and when these cells reached almost $80 \%$ confluence in the culture bottle, the previous medium was replaced with the serum-free medium, containing only DMEM/F12. Subsequently, the serum-free medium was collected after $24 \mathrm{~h}$ incubation, then centrifuged at 1,000 rpm for $5 \mathrm{~min}$, following which the supernatant was filtered with a $0.22 \mu \mathrm{m}$ syringe filter to acquire the A-CM and U-CM. The mediums were then conserved at $-20^{\circ} \mathrm{C}$ before use.

\section{CCK-8 cell proliferation assay}

The MCF-7 cells and MDA-MB-231 cells were digested by $0.25 \%$ trypsin when they reached over $90 \%$ confluence, then centrifuged at 1,000 rpm for $5 \mathrm{~min}$ and re-suspended 
Table 1 Sequences of oligomers and primers used

\begin{tabular}{ll}
\hline Name & Sequence $\left(5^{\prime}-3^{\prime}\right)$ \\
\hline Vimentin forward & GACCAGCTAACCAACGACAA \\
Vimentin reverse & GTTTTCGGCTTCCTCTCTCT \\
ATF4 forward & TTACAACCTCTTCCCCTTC \\
ATF4 reverse & CTTTATGCACTGAGGGATCA \\
MCL1 forward & TTGTCTCGAGTGATGATCCA \\
MCL1 reverse & CGAGAACGTCTGTGATACTTTC \\
BCL2 forward & TCATGTGTGTGGAGAGCGTC \\
BCL2 reverse & AGCCTCCGTTATCCTGGATC \\
18S forward & CCTGGATACCGCAGCTAGGA \\
18S reverse & GCGGCGCAATACGAATGCCCC \\
\hline
\end{tabular}

the cells. The cell concentrations were then adjusted to $5 \times 10^{4} / \mathrm{mL}$, and $100 \mu \mathrm{L}$ of the cell suspensions were added to the 96-well plates (each well with 5,000 cells). The $\mathrm{A}-\mathrm{CM}$ or $\mathrm{U}-\mathrm{CM}$ were then added $6 \mathrm{~h}$ later to replace the previous medium. After 12, 24, or $48 \mathrm{~h}$ incubation, the coculture medium was removed and replaced with $100 \mu \mathrm{L}$ fresh medium containing $10 \mu \mathrm{L}$ CCK- 8 , respectively, then incubated for $2 \mathrm{~h}$. Subsequently, the optical density (OD) value of each well were observed and analyzed by a scanning microplate spectrophotometer at $450 \mathrm{~nm}$ (A450). The experiment was performed three times.

\section{TUNEL assay}

For the TUNEL assay, apoptotic cells were detected using the in situ BrdU-Red DNA fragmentation (TUNEL) assay kit (Abcam, Cambridge, UK) and counterstained with 4',6'-diamidino-2-phenylindole hydrochloride (DAPI).

\section{Immunofluorescence assay}

The MCF-7 cells and MDA-MB-231 cells were collected and transferred into the 12 -well plates, respectively. When the cells were confluent, the previous medium was discarded and replaced with the A-CM or U-CM. After 12, 24 , or $48 \mathrm{~h}$ incubation, $4 \%$ paraformaldehyde was added to fix the cells for $15 \mathrm{~min}$, and the cells were washed three times with PBS. The cells were then incubated with $0.2 \%$ Triton X-100 for $1 \mathrm{~h}$ to increase the permeability of the cell membrane, then cultured at $4{ }^{\circ} \mathrm{C}$ and $1: 100$ dilution with the primary antibody Ki67, which is a nuclear antigen closely related to the progression of breast cancer. The cells were washed three times with PBS $24 \mathrm{~h}$ later, then incubated with FITC labeled goat anti-rabbit secondary antibody for $1 \mathrm{~h}$. Following this, DAPI was used to stain the cell nucleus for $15 \mathrm{~min}$. Finally, a Confocal Laser Scanning Microscope (CLSM) was used to observe the cells and analysis the fluorescence intensity.

\section{Real-time quantitative polymerase chain reaction (RT- $q P C R)$ assays}

Total RNA was obtained from the cell lines with TRIzol reagent (Invitrogen, CA, USA), and the concentration of RNA was measured by Nanodrop 2000c. Following the manufacturer's instructions, $500 \mathrm{ng}$ total RNA was then reverse transcribed to cDNA with a PrimeScript RT Master Mix (TaKaRa, Dalian, China) and quantitative realtime PCR was performed with SB Green Premix Ex Taq (TaKaRa, Dalian, China) reagent on the StepOnePlus system (Applied Biosystems, CA, USA). The internal control GAPDH. $2^{-\Delta \Delta C T}$ method was applied to calculate the fold changes. The primers used for the RT-qPCR assay are listed in Table 1.

\section{Western blot analysis}

Total proteins were extracted from the cell lines with RIPA lysis buffer (Beyotime Biotechnology, Shanghai, China) and the protease inhibitor phenylmethanesulfonyl fluoride (PMSF, 1:100, Beyotime). Bicinchoninic acid (BCA; Beyotime) reagent was then applied to determine the protein concentrations. Equal qualities of protein were then added to the sodium dodecyl sulfate polyacrylamide gel electrophoresis (SDS-PAGE) and transferred into polyvinylidene difluoride (PVDF) membranes (Millipore, MA, USA). To block the non-specific antigens, the PVDF membranes were incubated with $5 \%$ skimmed milk for $1 \mathrm{~h}$, followed by the primary antibodies (BCL2, MCL1, and GAPDH) at $4{ }^{\circ} \mathrm{C}$ overnight. After being washed with PBS three times, the membranes were then incubated with HRP-conjugated secondary antibody for $1 \mathrm{~h}$. Finally, an ECL detection system (FDbio, Shenzhen, China) was used to investigate the protein band.

\section{Statistical analysis}

All data were analyzed with IBM SPSS Statistics 20.0 (IBM, Chicago, IL, USA). Differences between two groups were 

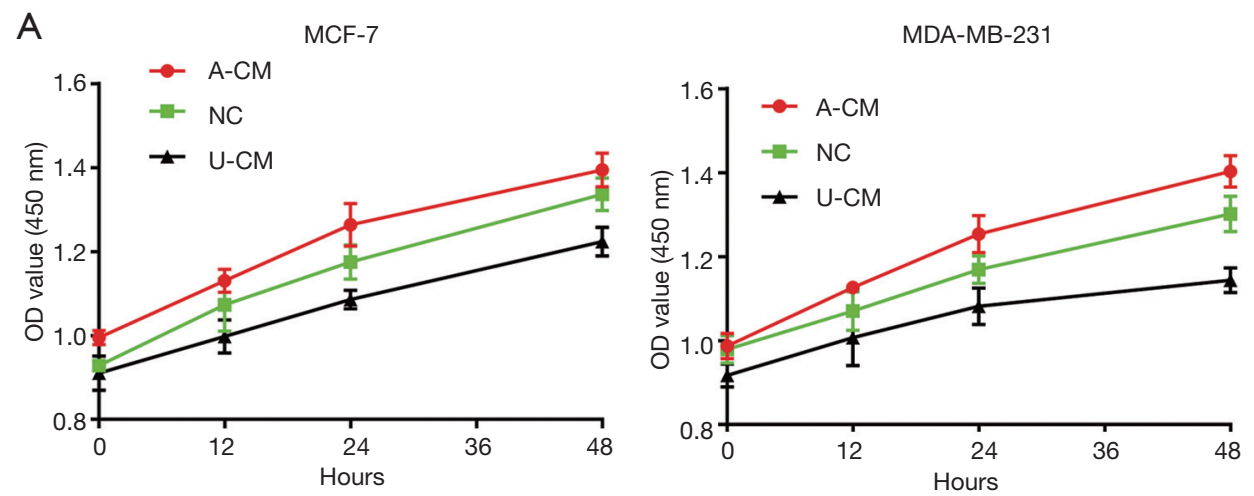

B
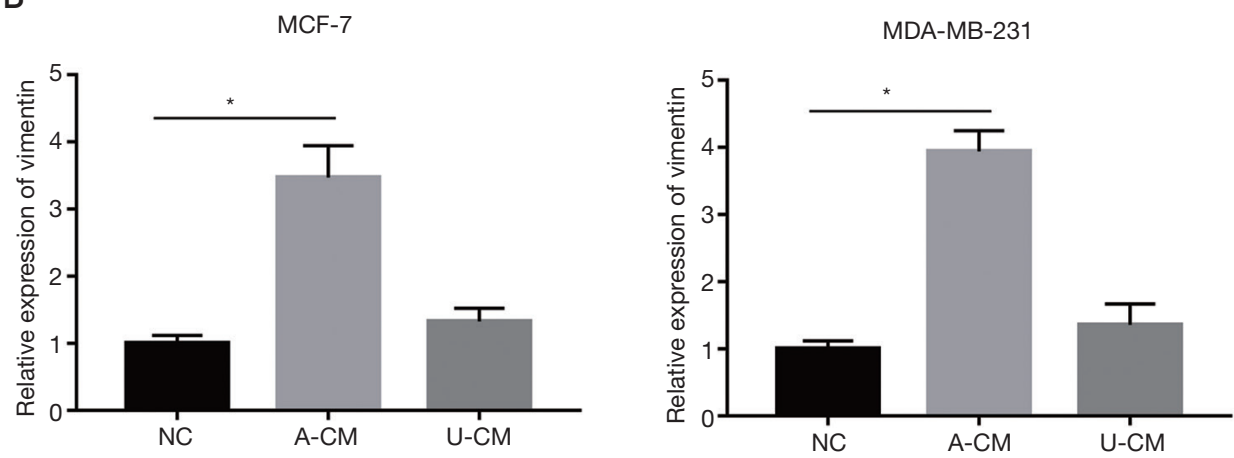

Figure 1 Cell proliferative ability of MCF-7 and MDA-MB-231 cells when co-cultured with A-CM or U-CM. (A) Viability of MCF-7 and MDA-MB-231 cells when treated with A-CM or U-CM, were detected by CCK-8 assay. (B) Relative expression of vimentin in MCF-7 and MDA-MB-231 cells at 12, 24, and $48 \mathrm{~h}$ under normal conditions. After incubation with A-CM or U-CM for $24 \mathrm{~h}$, the relative expression of vimentin in MCF-7 and MDA-MB-231 cells. (* indicates $\mathrm{P}<0.05$; NC: means natural control group, which were treated with free DMEM/ F12). ADSCs, adipose-derived stem cells; UMSCs, umbilical mesenchymal stem cells; A-CM, ADSCs-related medium; U-CM, UMSCsrelated medium; DMEM, Dulbecco's modified Eagle's medium; OD, optical density.

evaluated by an unpaired Student's $t$-test or analysis of variance (ANOVA), and a statistically significant difference was considered when $\mathrm{P}<0.05$. All experiments were repeated three times, unless otherwise indicated.

\section{Results}

\section{A-CM promoted cell proliferation for both MCF- 7 and MDA-MB-231 cells}

In vitro analysis with CCK-8 showed that A-CM remarkably promoted the proliferative capacity of MCF-7 and MDAMB-231 cells, while U-CM inhibited their growth (Figure 1A).

In addition, the growth promoting effects of MCF-7 and MDA-MB-231 cells with A-CM were confirmed by the results of RT-qPCR. Vimentin is a cytoskeletal protein closely associated with cell proliferation and the migration of cancer cells. Under normal conditions, the vimentin expression of MCF-7 and MDA-MB-231 cells is at a relatively low level (Figure 1B). However, after treatment with A-CM for $24 \mathrm{~h}$, the expression level of vimentin was more than twice that of the $\mathrm{NC}$ group $(\mathrm{P}<0.05$; Figure $1 B)$.

Moreover, a similar tendency was seen in the immunofluorescence assay, which showed the proportion of Ki67-positive cells was significantly higher in the A-CM group (Figure 2). Together, these results indicate that A-CM can promote MCF-7 and MDA-MB-231 cell growth in vitro, while U-CM inhibits their growth.

\section{The AC-M inbibited cell apoptosis for both MCF-7 and MDA-MB-231 cells}

The TUNEL assay showed the proportion of TUNELpositive cells was significantly higher in the A-CM and 
A

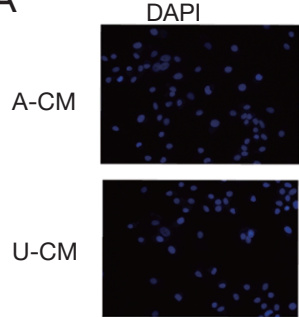

$\mathrm{NC}$

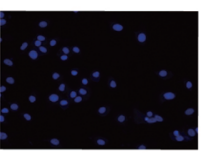

B
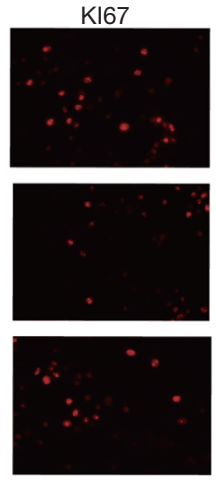

MCF-7
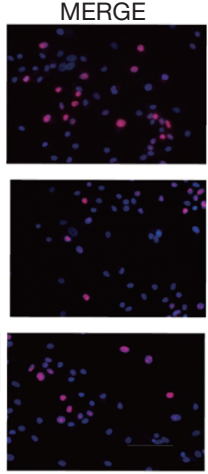

MCF-7

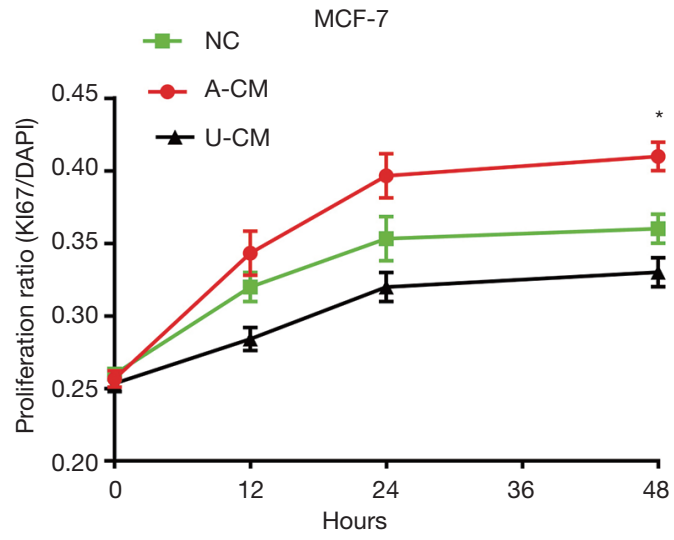

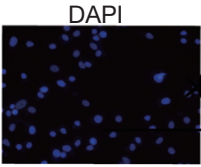
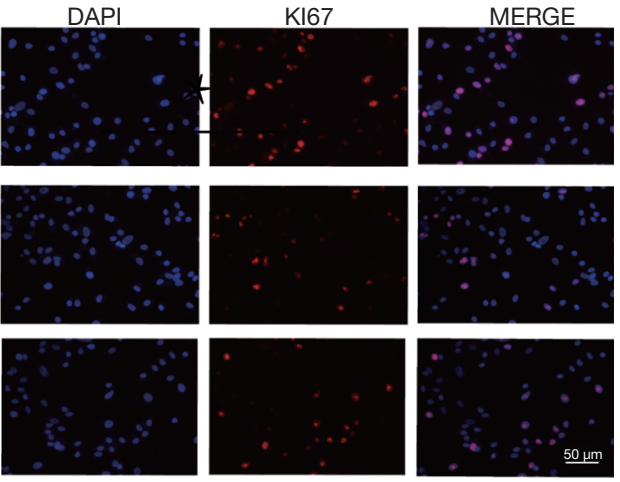

MDA-MB-231

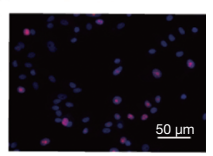

$50 \mu \mathrm{m}$

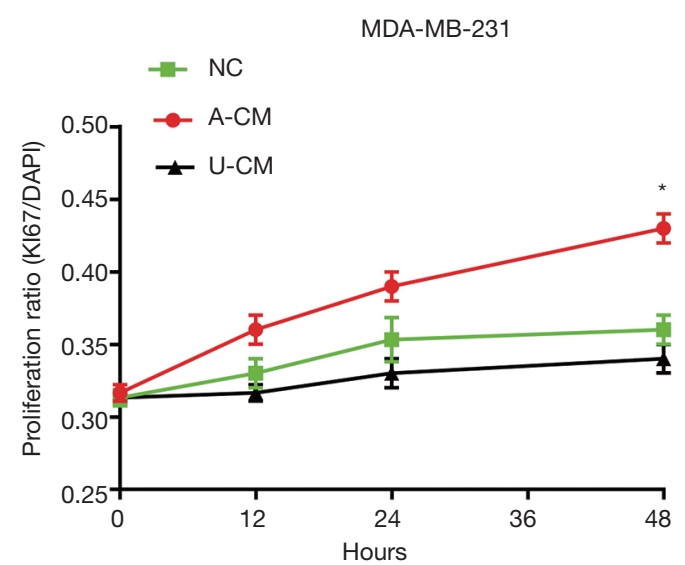

Figure 2 Immunofluorescence assay shows the proportion of Ki67-positive cells were significantly higher in the A-CM group. (A) Representative fluorescent picture of MCF-7 cells and MDA-MB-231 cells at $48 \mathrm{~h}$ of incubation with A-CM or U-CM (upper; magnification, 400×). (B) Corresponding quantification using the blue mean fluorescence intensity of DAPI at 12, 24, and 48 h of incubation with A-CM or U-CM (lower). (* indicates $\mathrm{P}<0.05$; NC: means natural control group, which were treated with free DMEM/F12). ADSCs, adipose-derived stem cells; UMSCs, umbilical mesenchymal stem cells; A-CM, ADSCs-related medium; U-CM, UMSCs-related medium; DAPI, 4',6'-diamidino-2-phenylindole hydrochloride; DMEM, Dulbecco's modified Eagle's medium.

U-CM groups for MCF-7, while only slightly higher in the A-CM group for MDA-MB-231 (Figure 3). These results indicate that A-CM could inhibit MCF-7 and MDAMB-231 cell apoptosis in vitro, while U-CM could only inhibit MCF-7.

\section{Co-culture of A-CM up-regulates mRNA expression of ATF4 and its downstream genes (MCL1 and BCL2) in MCF- 7 and MDA-MB-231 cells}

The observation that A-CM and U-CM exerted contrary effect on the proliferation of MCF-7 and MDA-MB-231 cells caused us to search for a mechanism to explain this result, and a RT-qPCR analysis was performed to determine the mRNA expression level of ATF4, MCL1, and BCL2. As shown in Figure 4, compared with the NC group and U-CM group, the expression level of ATF4 was remarkably up-regulated after being co-cultured with A-CM for $24 \mathrm{~h}$ (Figure $4 A$ ). However, we also found that in the A-CM group, the mRNA of BCL2 (Figure $4 B$ ) and MCL1 (Figure $4 C$ ) were over-expressed relative to the $\mathrm{NC}$ group.

\section{Co-culture of A-CM down-regulates protein expression of MCL1 and BCL2 in MCF-7 and MDA-MB-231 cells}

To further investigate the effects of A-CM and U-CM in 

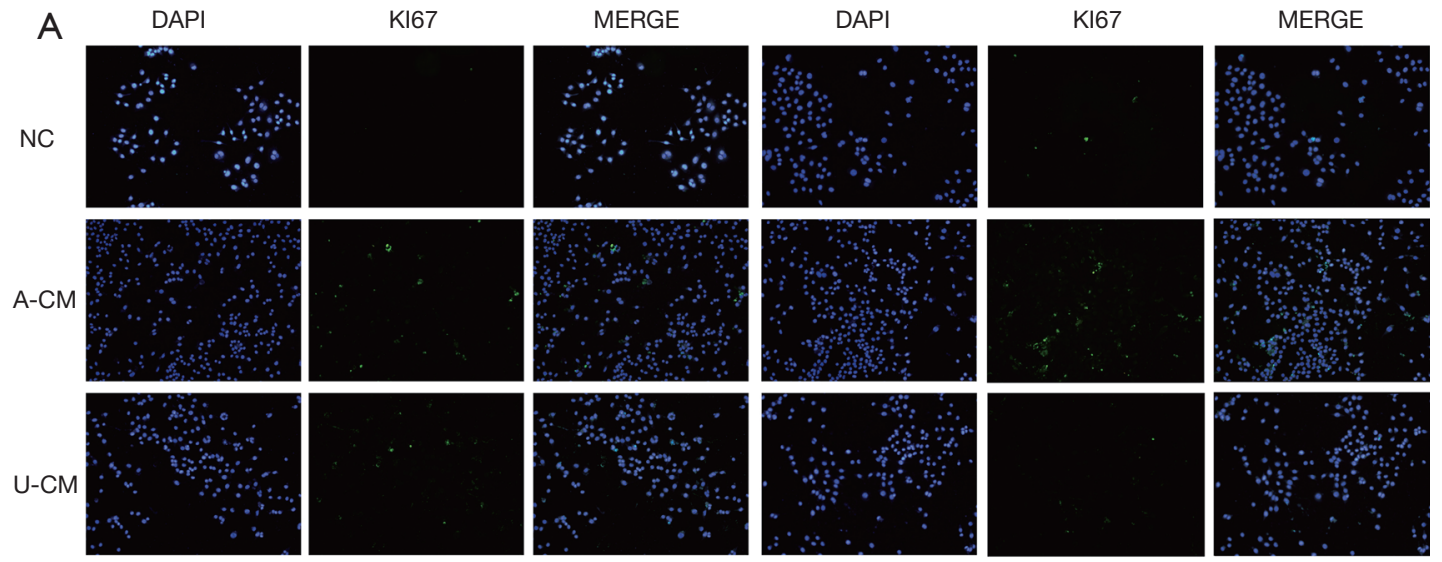

B

MCF-7
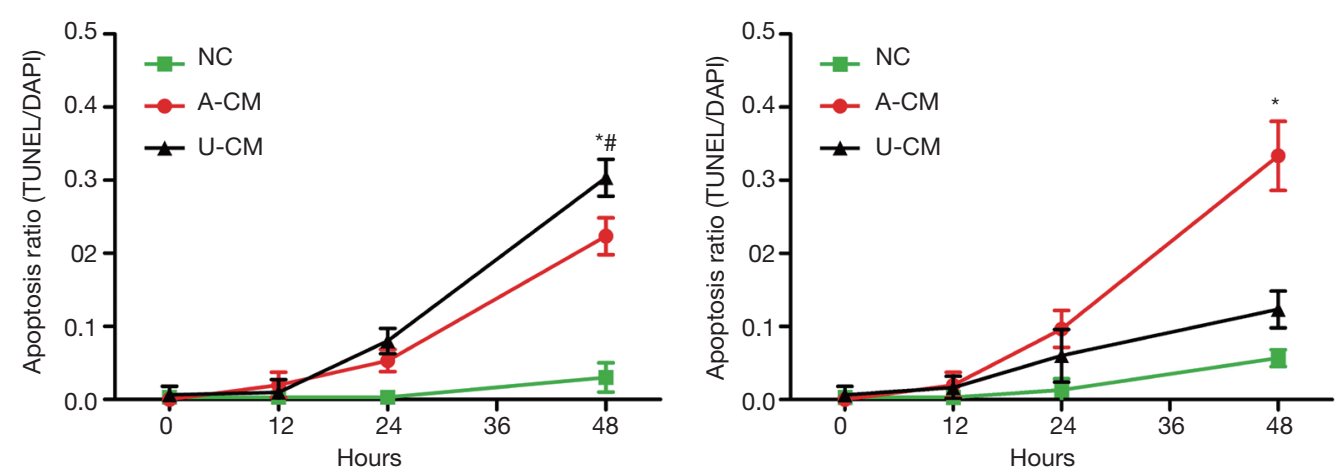

Figure 3 Apoptosis assay shows the proportion of TUNEL-positive cells were significantly higher in the A-CM group. (A) Representative fluorescent picture of MCF-7 cells and MDA-MB-231 cells at $48 \mathrm{~h}$ of incubation with A-CM or U-CM (upper; magnification, 200×). (B) Corresponding quantification using the blue mean fluorescence intensity of DAPI at 12, 24, and $48 \mathrm{~h}$ of incubation with A-CM or U-CM (lower). (* indicates $\mathrm{P}<0.05, \mathrm{~A}-\mathrm{CM}$ vs. NC; ${ }^{*}$ indicates $\mathrm{P}<0.05$, U-CM vs. NC; NC: means natural control group, which were treated with free DMEM/F12). ADSCs, adipose-derived stem cells; UMSCs, umbilical mesenchymal stem cells; A-CM, ADSCs-related medium; U-CM, UMSCs-related medium; DAPI, 4',6'-diamidino-2-phenylindole hydrochloride; DMEM, Dulbecco's modified Eagle's medium.

MCF-7 and MDA-MB-231 cells, Western blot was applied to analyze the anti-apoptosis protein (MCL1 and BCL2) expression (Figure 5A). Interestingly, we discovered that in contrast to the results of the RT-qPCR, both A-CM and $\mathrm{U}-\mathrm{CM}$ could inhibit the expression of MCL1 and BCL2 proteins (Figure 5). Thus, although the mRNA expression level of anti-apoptosis genes (MCL1 and BCL2) were upregulated, A-CM could inhibit the translation process of MCL1 and BCL2 mRNA, then promote apoptosis of MCF7 and MDA-MB-231 cells.

\section{Discussion}

Our results demonstrated that the A-CM could interfere with the translation process of MCL1 and BCL2 mRNA and inhibit the expression of MCL1 and BCL2 proteins through upregulation of ATF4, inducing cell apoptosis. These data provide insight into the safety characteristics of AFG.

While AFG has been widely applied in clinical use for breast reconstruction, its safety for breast cancer patients remained unclear $(5,6)$. A growing body of basic research indicates that after treatment with AFG, ADSCs secrete cell factors such as adipsin (20) and leptin (21) which promote the tumor micro-environment for breast cancer cell growth and increase the risk of breast cancer recurrence. While these studies did raise concerns for the clinical use of AFG for breast reconstruction, clinical trials have since indicated that there was no significant difference in recurrence rate 
A

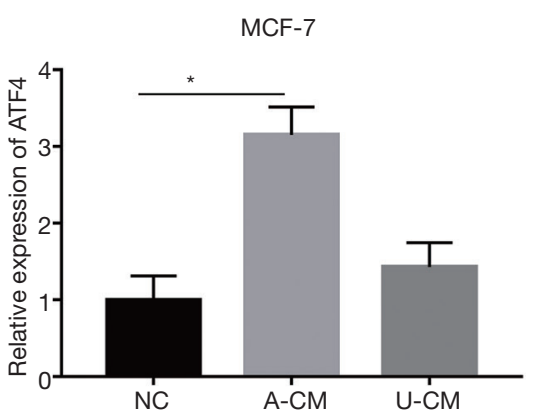

B

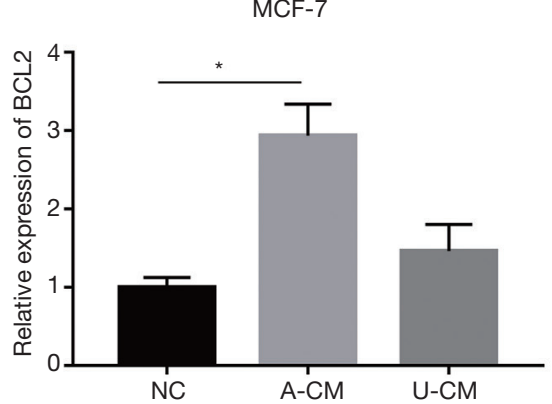

C

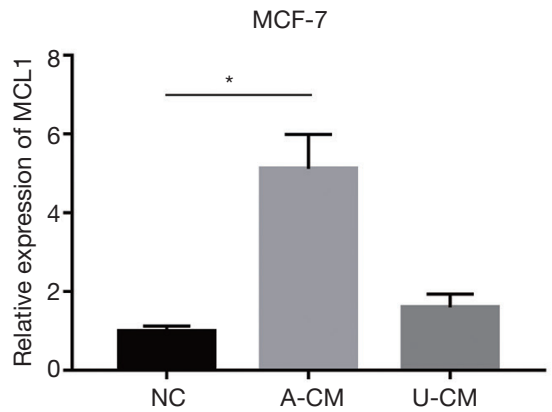

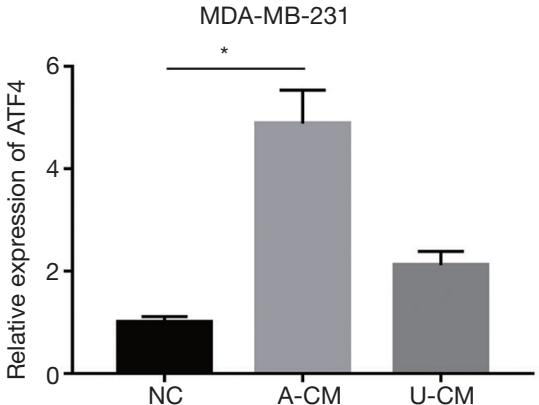
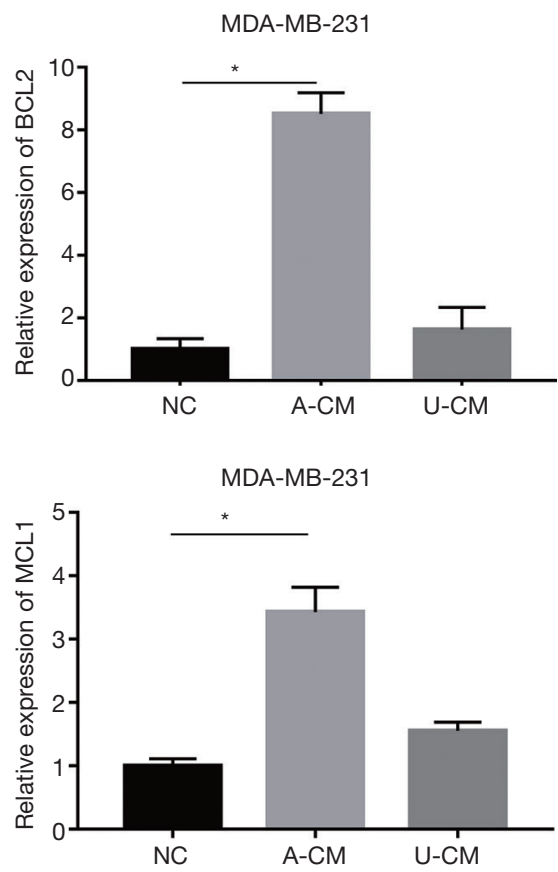

Figure 4 A-CM up-regulated the expression of ATF4 (A), BCL2 (B), and MCL1 (C) mRNA in MCF-7 and MDA-MB-231 cells. Expression level of ATF4, BCL2, and MCL1 mRNA, after MDA-MB-231 cells were co-cultured with A-CM or U-CM for $24 \mathrm{~h}$. ( ${ }^{*}$ indicates $\mathrm{P}<0.05$; NC: means natural control group, which were treated with free DMEM/F12). ADSCs, adipose-derived stem cells; UMSCs, umbilical mesenchymal stem cells; A-CM, ADSCs-related medium; U-CM, UMSCs-related medium; ATF4, activating transcription factor 4; DMEM, Dulbecco's modified Eagle's medium.

and the progression of breast cancer $(12,22)$. Consistent with the results of clinical trials, another study constructed a novel mouse model with AFG treatment and confirmed that ADSCs and their secretions did not promote tumor growth (23). Therefore, to improve the prognosis of breast cancer patients to the utmost, it was important to explore the underlying mechanisms of the contradictory results between basic studies and clinical studies.

In the present study, MCF-7 and MDA-MB-231 breast cancer cells were treated with A-CM or U-CM. The results of CCK-8 assay, immunofluorescence assay, and the vimentin mRNA expression showed that compared with the control group, A-CM could promote breast cancer cell growth while U-CM did not, which is consistent with the results of previous basic research. However, this failed to explain the results from clinical trials, so we further explored the relationship between A-CM and the expression of ATF4, and the RT-qPCR results showed that ATF4 was up-regulated in MCF-7 and MDA-MB-231 cells when cocultured with the A-CM. ATF4 is an important intracellular signaling molecule involved in the procession and development of various tumors and could induce apoptosis 
A

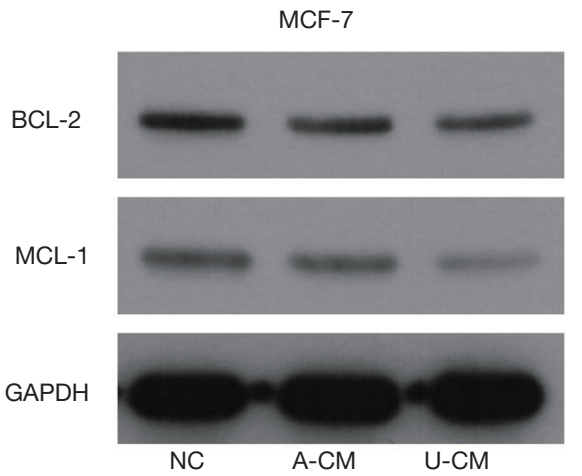

B

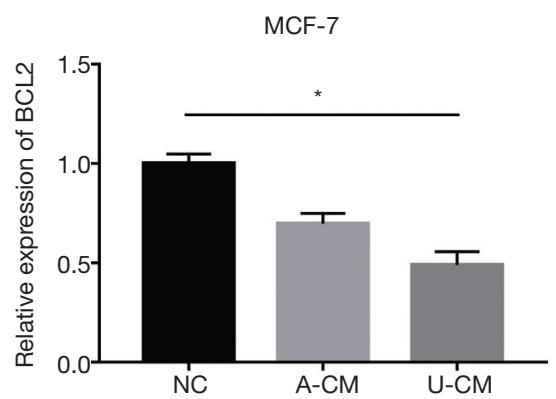

C

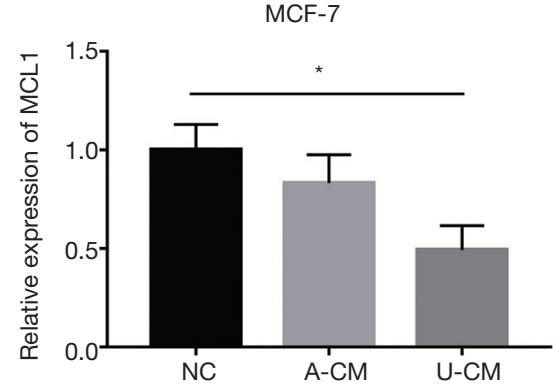

MDA-MB-231
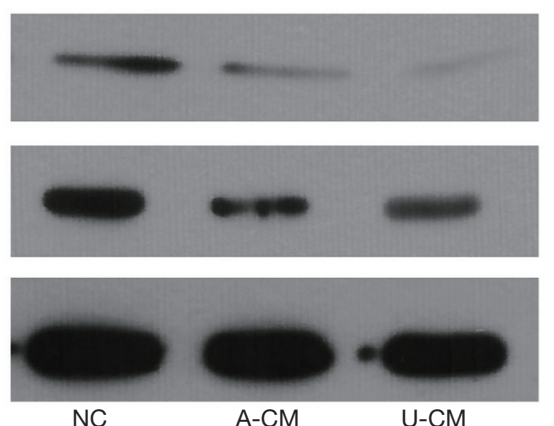

NC
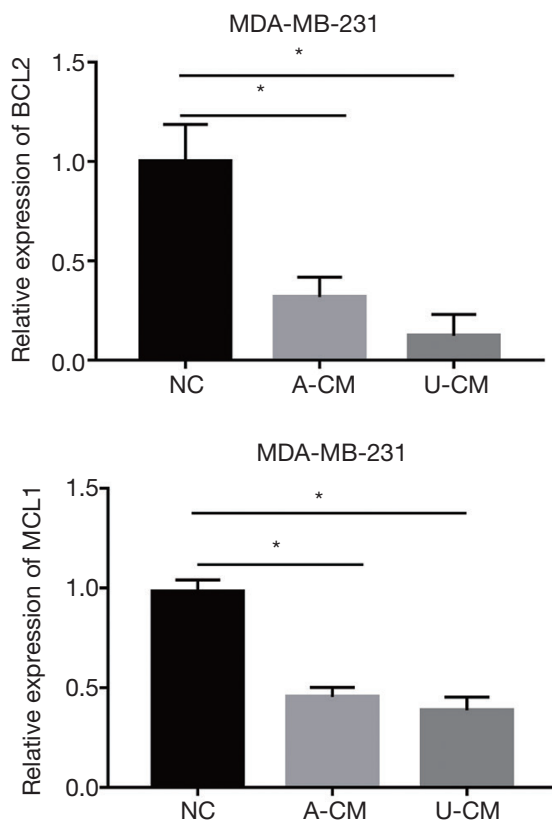

Figure 5 Both A-CM and U-CM down-regulated the expression of anti-apoptosis proteins (BCL2 and MCL1) in MCF-7 and MDAMB-231 cells. (A) Expression level of MCL1 and BCL2 protein in MCF-7 cells, after co-culture with A-CM or U-CM for 48 h. (B,C) Expression level of BCL2 and MCL1 protein in MDA-MB-231 cells, after co-culture with A-CM or U-CM for 48 h. ( ${ }^{*}$ indicates $\mathrm{P}<0.05$; NC: means natural control group, which were treated with free DMEM/F12). ADSCs, adipose-derived stem cells; UMSCs, umbilical mesenchymal stem cells; A-CM, ADSCs-related medium; U-CM, UMSCs-related medium; DMEM, Dulbecco's modified Eagle's medium.

(24,25). Moreover, the Western blot results showed that the anti-apoptosis proteins (MCL1 and BCL2) were downregulated, which meant that A-CM could induce cell apoptosis through ATF4. However, interestingly, we found that the mRNA expression of anti-apoptosis gene (MCL1 and BCL2) was increased, which was in contradiction with the Western blot results. Further research is required to explain this apparent paradox.

Previous studies have shown that A-CM could inhibit cell apoptosis via the PI3K/Akt pathway (26,27). Consequently, when breast cancer cells were co-cultured with the ADSCs, A-CM could up-regulate the expression of the anti-apoptosis gene, and its mRNA expression increased. However, the over-expression of ATF4 would interfere with the translation process of MCL1 and BCL2 mRNA through the following pathway: the over-expression of ATF4 is closely associated with the phosphorylation of eIF $2 \alpha$, which could negatively regulate the translation level of the gene $(28,29)$. In addition, ATF4 could enter the cell nucleus and promote the expression of the $\mathrm{C} / \mathrm{EBP}-\mathrm{homologous}$ protein 
(CHOP) (19). As a typical pro-apoptotic transcription factor, CHOP could inhibit anti-apoptosis protein expression and induce cell apoptosis $(30,31)$. Together, this may explain how A-CM could induce cell apoptosis through the eIF2 $\alpha / \mathrm{ATF} 4 / \mathrm{CHOP}$ pathway.

\section{Conclusions}

The results of this study indicate that contrary to previous basic research findings, because A-CM promotes cell apoptosis, it is reasonable to assume that AFG does not promote tumor growth and is safe for breast cancer patients.

\section{Acknowledgments}

Funding: This study was supported by the National Nature Science Foundation of China (grant No. 81601702, 81671931, 81772101, 81701920, 81801933, 81801932, $81871573,81901976,81901975$ and 81971852), Natural Science Foundation of Guangdong Province of China (grant No. 2021A1515011623), the Medical Scientific Research Foundation of Guangdong Province of China (grant No. A2020542, A2021446), the Administrator Foundation of Nanfang Hospital (grant No. 2019B021, 2020Z004) and the National Undergraduate Innovation and Entrepreneurship Training Program (grant No. X202012121222, X202012121312).

\section{Footnote}

Reporting Checklist: The authors have completed the MDAR reporting checklist. Available at https://dx.doi. org/10.21037/atm-21-3746

Data Sharing Statement: Available at https://dx.doi. org/10.21037/atm-21-3746

Conflicts of Interest: All authors have completed the ICMJE uniform disclosure form (available at https://dx.doi. org/10.21037/atm-21-3746). The authors have no conflicts of interest to declare.

Ethical Statement: The authors are accountable for all aspects of the work in ensuring that questions related to the accuracy or integrity of any part of the work are appropriately investigated and resolved.

Open Access Statement: This is an Open Access article distributed in accordance with the Creative Commons Attribution-NonCommercial-NoDerivs 4.0 International License (CC BY-NC-ND 4.0), which permits the noncommercial replication and distribution of the article with the strict proviso that no changes or edits are made and the original work is properly cited (including links to both the formal publication through the relevant DOI and the license). See: https://creativecommons.org/licenses/by-nc-nd/4.0/.

\section{References}

1. Bray F, Ferlay J, Soerjomataram I, et al. Global cancer statistics 2018: GLOBOCAN estimates of incidence and mortality worldwide for 36 cancers in 185 countries. CA Cancer J Clin 2018;68:394-424. Erratum in: CA Cancer J Clin 2020;70:313.

2. Coleman SR, Saboeiro AP. Fat grafting to the breast revisited: safety and efficacy. Plast Reconstr Surg 2007;119:775-85; discussion 786-7.

3. Delay E, Garson S, Tousson G, et al. Fat injection to the breast: technique, results, and indications based on 880 procedures over 10 years. Aesthet Surg J 2009;29:360-76.

4. Mizuno H, Hyakusoku H. Fat grafting to the breast and adipose-derived stem cells: recent scientific consensus and controversy. Aesthet Surg J 2010;30:381-7.

5. Claro F Jr, Figueiredo JC, Zampar AG, et al. Applicability and safety of autologous fat for reconstruction of the breast. Br J Surg 2012;99:768-80.

6. Gutowski KA; ASPS Fat Graft Task Force. Current applications and safety of autologous fat grafts: a report of the ASPS fat graft task force. Plast Reconstr Surg 2009;124:272-80.

7. Wang YY, Lehuédé $\mathrm{C}$, Laurent $\mathrm{V}$, et al. Adipose tissue and breast epithelial cells: a dangerous dynamic duo in breast cancer. Cancer Lett 2012;324:142-51.

8. Park J, Euhus DM, Scherer PE. Paracrine and endocrine effects of adipose tissue on cancer development and progression. Endocr Rev 2011;32:550-70.

9. Yoshimura K, Sato K, Aoi N, et al. Cell-assisted lipotransfer for cosmetic breast augmentation: supportive use of adipose-derived stem/stromal cells. Aesthetic Plast Surg 2008;32:48-55; discussion 56-7.

10. Wang Y, Liu J, Jiang Q, et al. Human adipose-derived mesenchymal stem cell-secreted CXCL1 and CXCL8 facilitate breast tumor growth by promoting angiogenesis. Stem Cells 2017;35:2060-70.

11. Duong MN, Cleret A, Matera EL, et al. Adipose cells promote resistance of breast cancer cells to trastuzumab- 


\section{Page 10 of 10}

mediated antibody-dependent cellular cytotoxicity. Breast Cancer Res 2015;17:57.

12. Krastev T, van Turnhout A, Vriens E, et al. Long-term follow-up of autologous fat transfer vs conventional breast reconstruction and association with cancer relapse in patients with breast cancer. JAMA Surg 2019;154:56-63.

13. Brown AWW, Kabir M, Sherman KA, et al. Patient reported outcomes of autologous fat grafting after breast cancer surgery. Breast 2017;35:14-20.

14. Agha RA, Fowler AJ, Herlin C, et al. Use of autologous fat grafting for breast reconstruction: a systematic review with meta-analysis of oncological outcomes. J Plast Reconstr Aesthet Surg 2015;68:143-61.

15. De Decker M, De Schrijver L, Thiessen F, et al. Breast cancer and fat grafting: efficacy, safety and complications-a systematic review. Eur J Obstet Gynecol Reprod Biol 2016;207:100-8.

16. Tang YM, Bao WM, Yang JH, et al. Umbilical cordderived mesenchymal stem cells inhibit growth and promote apoptosis of HepG2 cells. Mol Med Rep 2016;14:2717-24.

17. Ciavarella S, Caselli A, Tamma AV, et al. A peculiar molecular profile of umbilical cord-mesenchymal stromal cells drives their inhibitory effects on multiple myeloma cell growth and tumor progression. Stem Cells Dev 2015;24:1457-70.

18. Wei N, Zhu LQ, Liu D. ATF4: a novel potential therapeutic target for Alzheimer's disease. Mol Neurobiol 2015;52:1765-70.

19. Wang X, Xia HY, Qin HY, et al. 20(S)-protopanaxadiol induces apoptosis in human umbilical vein endothelial cells by activating the PERK-eIF2alpha-ATF4 signaling pathway. J Cell Biochem 2019;120:5085-96.

20. Goto H, Shimono Y, Funakoshi Y, et al. Adipose-derived stem cells enhance human breast cancer growth and cancer stem cell-like properties through adipsin. Oncogene 2019;38:767-79.

21. Delle Monache S, Calgani A, et al. Adipose-derived stem cells sustain prolonged angiogenesis through leptin

Cite this article as: Ren J, Kong W, Lu F, Li Y. Adiposederived stem cells (ADSCs) inhibit the expression of antiapoptosis proteins through up-regulation of ATF4 on breast cancer cells. Ann Transl Med 2021;9(16):1300. doi: 10.21037/ atm-21-3746
Ren et al. ADSCs inhibit anti-apoptosis proteins on breast cancer

secretion. Growth Factors 2016;34:87-96.

22. Mazur S, Zołocińska A, Siennicka K, et al. Safety of adipose-derived cell (stromal vascular fraction - SVF) augmentation for surgical breast reconstruction in cancer patients. Adv Clin Exp Med 2018;27:1085-90.

23. Tsuji W, Valentin JE, Marra KG, et al. An animal model of local breast cancer recurrence in the setting of autologous fat grafting for breast reconstruction. Stem Cells Transl Med 2018;7:125-34.

24. Fan CF, Miao Y, Lin XY, et al. Expression of a phosphorylated form of ATF4 in lung and non-small cell lung cancer tissues. Tumour Biol 2014;35:765-71.

25. Zhu H, Chen X, Chen B, et al. Activating transcription factor 4 promotes esophageal squamous cell carcinoma invasion and metastasis in mice and is associated with poor prognosis in human patients. PLoS One 2014;9:e103882.

26. Zhang J, Zhou S, Zhou Y, et al. Adipose-derived mesenchymal stem cells (ADSCs) with the potential to ameliorate platelet recovery, enhance megakaryopoiesis, and inhibit apoptosis of bone marrow cells in a mouse model of radiation-induced thrombocytopenia. Cell Transplant 2016;25:261-73.

27. Yu X, Su B, Ge P, et al. Human adipose derived stem cells induced cell apoptosis and $\mathrm{s}$ phase arrest in bladder tumor. Stem Cells Int 2015;2015:619290.

28. Hetz C. The unfolded protein response: controlling cell fate decisions under ER stress and beyond. Nat Rev Mol Cell Biol 2012;13:89-102.

29. Harding HP, Novoa I, Zhang Y, et al. Regulated translation initiation controls stress-induced gene expression in mammalian cells. Mol Cell 2000;6:1099-108.

30. Doyle KM, Kennedy D, Gorman AM, et al. Unfolded proteins and endoplasmic reticulum stress in neurodegenerative disorders. J Cell Mol Med 2011;15:2025-39.

31. Toth A, Nickson P, Mandl A, et al. Endoplasmic reticulum stress as a novel therapeutic target in heart diseases. Cardiovasc Hematol Disord Drug Targets 2007;7:205-18. 